\title{
EVOLUTION OF 1-5 $\mathrm{M}_{\odot}$ STARS WITH MASS LOSS
}

\author{
E. VASSILIADIS and P.R. WOOD \\ Mount Stromlo and Siding Spring Observatories, Private Bag, Weston Creek P.O., Canberra, \\ ACT 2611, Australia
}

Stars of mass $1-5 \mathrm{M}_{\odot}$ and composition $Y=0.25$ and $Z=0.016$ have been evolved from the main-sequence to the white dwarf stage with an empirical mass loss formula based on observations of mass loss rates in AGB stars. This mass loss formula (Wood 1990) causes the mass loss rate to rise exponentially with pulsation period on the AGB until superwind rates are achieved, where these rates correspond to radiation pressure driven mass loss rates. The formula was designed to reproduce the maximum periods observed for optically-visible LPVs and it also reproduces extremely well the maximum AGB luminosities observed in star clusters in the Magellanic Clouds (see Vassiliadis and Wood 1992 for details).

During AGB evolution, deep envelope convection exits in the $5 \mathrm{M}_{\odot}$ star while the envelope mass is $>1.6 \mathrm{M}_{\bullet}$. The deep envelope convection has several consequence: (1) the hydrogenexhausted core mass $M_{c}$ is effectively constant with time, since the increase in $\mathbf{M}_{c}$ during the hydrogen-burning phases is eliminated at the next shell flash when almost all the burnt hydrogen is dredged up by envelope convection $(\lambda \approx 1)$, (2) the burning of the helium shell at a shell flash is quenched very rapidly by the cooling effect of the deep envelope convection, so that the helium exhausted core mass does not increase significantly with time, and (3) shell flashes have very little effect on the surface luminosity because of the small amount of energy released by helium burning and the quick recovery of hydrogen burning.

When mass loss has reduced the envelope mass below $\sim 1.6 \mathrm{M}_{\bullet}$, convective penetration by the envelope is no longer deep and 'normal' helium shell flash behaviour returns - the core mass increases with time, the hydrogen and helium shells burn outward at the same average rate, and the surface luminosity varies by large amounts during the shell flash cycle.

The $5 \mathrm{M}_{\odot}$ star produces a $0.90 \mathrm{M}_{\odot}$ remnant which evolves through the planetary nebula region of the HR diagram very rapidly until $\log \mathrm{L} / \mathrm{L}_{\odot}$ has declined to $\sim 2.5$ where the evolution begins to slow down, in agreement with the calculations of the evolution of massive PNN by Paczynski (1971) and Faulkner and Wood (1986). The results contrast with those of Blöcker and Schönberner (1990) who find a $0.836 \mathrm{M}_{\odot}$ remnant which is $\sim 3$ times more luminous at an age of $10^{4}$ years than similar models from our calculations.

\section{References}

Blöcker, T. and Schönberner, D. 1990, A\&AL, 240, L11.

Paczynski, B. 1971, Acta Astr., 21, 417.

Vassiliadis, E. and Wood, P.R. 1992, in preparation.

Wood, P.R. 1990, in From Miras to Planetary Nebulae, eds. M.O. Mennessier and A. Omont (Editions Frontières), p. 67.

Wood, P.R. and Faulkner, D.J. 1986, ApJ, 307, 659. 